\title{
Quantum state engineering by nondeterministic noiseless linear amplification
}

\author{
Hamza Adnane, ${ }^{1}$ Matteo Bina, ${ }^{2}$ Francesco Albarelli, ${ }^{3}$ Abdelhakim Gharbi, ${ }^{1}$ and Matteo G. A. Paris ${ }^{2}$ \\ ${ }^{1}$ Laboratoire de Physique Théorique, Faculté des Sciences Exactes, Université de Bejaia, 06000 Bejaia, Algeria \\ ${ }^{2}$ Quantum Technology Lab, Dipartimento di Fisica, Università degli Studi di Milano, I-20133 Milano, Italy \\ ${ }^{3}$ Department of Physics, University of Warwick, Coventry CV4 7AL, United Kingdom
}

(Received 31 January 2019; revised manuscript received 4 April 2019; published 18 June 2019)

\begin{abstract}
We address quantum state engineering of single- and two-mode states by means of nondeterministic noiseless linear amplifiers (NLAs) acting on Gaussian states. In particular, we consider the optimal implementation of a NLA proposed by Pandey et al. [Phys. Rev. A 88, 033852 (2013)] and we show that it provides an effective scheme to generate highly non-Gaussian and nonclassical states, when used outside the regime of high-fidelity amplification. Additionally, we show that in this regime also the amplification of a two-mode squeezed vacuum state (twin-beam) highly increases entanglement.
\end{abstract}

DOI: 10.1103/PhysRevA.99.063823

\section{INTRODUCTION}

Nonclassical and non-Gaussian states of continuousvariable systems have been a relevant resource in the development of quantum information science and technology, as well as in several fundamental tests of quantum mechanics itself [1-3]. Quantum state engineering of those states, however, is often hampered by two major challenges. On the one hand, generation of nonclassical light usually involves nonlinear optical media, and the small value of nonlinear susceptibilities leads to low efficiency. On the other hand, light amplification is usually involved as well and the linearity and unitarity of quantum dynamics make this task rather difficult, since they impose that noise should be unavoidably added when a signal is amplified, in order to maintain the uncertainty relation [4].

Recently, in order to circumvent these difficulties, amplifying devices have been suggested, which act nondeterministically, i.e., the output state is obtained conditionally by postselecting on a particular measurement outcome [5-12]. In particular, optimal schemes describing nondeterministic linear amplifiers (NLAs) achieving successful amplification with the largest probability allowed by quantum mechanics have been put forward in theoretical papers [13,14]. These kinds of devices are appealing for quantum state engineering, especially in the continuous-variable regime, where several schemes based on conditional states of continuous-variable measurements have been already explored and proved effective [15-22].

In this paper, we address quantum state engineering of single- and two-mode states aided by the optimal nondeterministic noiseless linear amplifier introduced in [13], acting on Gaussian states. In particular, we prove that such a NLA provides an effective scheme to generate highly non-Gaussian and nonclassical states. Crucially, we distinguish two working regimes for this kind of NLA. When the device acts on an input state that lies in a phase-space disk of radius $\sqrt{N} / g$ centered at the origin, such a device generates an approximately ideal amplified state with the highest possible fidelity [13]. On the other hand, in the remaining region, where no constraints are imposed to the fidelity, the NLA proves to be a robust resource for non-Gaussianity and nonclassicality.

We provide a general framework to address state engineering by NLA, and present explicit results for the nonGaussianity and nonclassicality obtained with single-mode coherent and squeezed vacuum states, as well as with twomode squeezed vacuum (twin-beam state). In order to assess quantitatively the performances of NLAs on those signals, we quantify nonclassicality using negativity of the Wigner function (from now on W nonclassicality) [23] and nonGaussianity by the relative entropy to a reference Gaussian state $[24,25]$. We remark that these quantifiers have recently been studied also in the context of quantum resource theories [26-29].

Concerning the action of the NLA on two-mode states, we consider two scenarios. In the first one, we focus on the performances of a strong (destructive) NLA measurement on one mode of a twin beam and analyze the properties of the resulting single-mode conditional states [18]. Our results show that, depending on the two-mode squeezing parameter and the gain of the device, the resulting conditional state has a Wigner function that includes negative parts. This suggests that such a scheme represents a robust source for nonclassicality. The second regime that we explore is the proper (nondestructive) action of the NLA on one mode of the twin-beam state. Here, we address the degree of entanglement of the amplified state as a function of the NLA gain parameter, and prove that it may be significantly enhanced. This would be expected in the high-fidelity region, where the amplifier acts closely to an ideal one by increasing the two-mode squeezing parameter. Nonetheless, we show that also in the other regime the proposed device still enhances the entanglement by making the state non-Gaussian.

The paper is organized as follows. In Sec II, we establish notation and review the nonclassicality and non-Gaussianity measures used across this work, that is, the W nonclassicality and the entropic non-Gaussianity. We also recall the main ingredients needed to describe nondeterministic linear amplifiers. In Sec. III, we discuss quantum state engineering 
by NLA on coherent and squeezed vacuum states, focusing our attention on nonclassicality and non-Gaussianity of the conditionally amplified states. Section IV is devoted to conditional states generation by exploiting the action of NLAs on twin beam, in both the destructive and nondestructive regimes. Section V closes the paper with concluding remarks.

\section{PRELIMINARIES}

In this section, we briefly introduce the tools we will use throughout the paper to quantify non-Gaussianity (nG) and nonclassicality (nC) of a single-mode bosonic quantum state. For the characterization of $\mathrm{nG}$ we employ a measure based on the quantum relative entropy (QRE) between the state under examination and a reference Gaussian state $[24,25]$. Concerning the $\mathrm{nC}$, we use an indicator based on the volume of the negative part of the Wigner function [23]. The final subsection is devoted to briefly reviewing the main results about the measurement operators of a noiseless linear amplifier, as well as about the corresponding conditionally amplified states [14].

\section{A. Non-Gaussianity based on quantum relative entropy}

In continuous-variable systems, a single-mode radiation field is described by creation and annihilation operators, $\hat{a}^{\dagger}$ and $\hat{a}$, respectively, satisfying the bosonic commutation relation $\left[\hat{a}, \hat{a}^{\dagger}\right]=\hat{\mathbb{I}}$. Coherent states are the eigenstates of the annihilation operator $|\alpha\rangle$, such that $\hat{a}|\alpha\rangle=\alpha|\alpha\rangle$, whereas number states $|n\rangle$ are the eigenstates of the number operator $\hat{a}^{\dagger} \hat{a}|n\rangle=n|n\rangle$. The operators $\hat{x}=\left(\hat{a}+\hat{a}^{\dagger}\right) / \sqrt{2}$ and $\hat{p}=$ $i\left(\hat{a}^{\dagger}-\hat{a}\right) / \sqrt{2}$ describe the observable quadratures of the field. A quantum state, i.e., a density operator $\hat{\varrho}$, may be represented in the phase space by means of the characteristic function $\chi[\hat{\varrho}](\alpha)=\operatorname{Tr}[\hat{\varrho} \hat{D}(\alpha)]$, where $\hat{D}(\alpha)=\exp \left\{\alpha \hat{a}^{\dagger}-\alpha^{*} \hat{a}\right\}$ is the displacement operator. The Wigner function, defined as the Fourier transform of the characteristic function $W[\hat{\varrho}](\lambda) \propto$ $\int d^{2} \alpha \exp \left\{\lambda^{*} \alpha-\lambda \alpha^{*}\right\} \chi[\hat{\varrho}](\alpha)$, is the most iconic quasiprobability distribution for the quantum state [30]. In particular, it is the only one in the family of the $p$-ordered quasiprobability distributions, widely used in quantum optics [31,32], that gives the probability densities for quadrature measurements as its marginal distributions.

Gaussian states [33-36] are quantum states having a Gaussian Wigner function

$$
W\left[\hat{\varrho}_{G}\right](\mathbf{X})=\frac{\exp \left[-\frac{1}{2}(\mathbf{X}-\langle\hat{\mathbf{R}}\rangle)^{T} \boldsymbol{\sigma}^{-1}(\mathbf{X}-\langle\hat{\mathbf{R}}\rangle)\right]}{2 \pi \sqrt{\operatorname{det} \boldsymbol{\sigma}}},
$$

where we considered the Cartesian representation of real variables $\boldsymbol{X}=(x, p)^{T}$. A Gaussian state is fully identified by its first-moment vector and its covariance matrix (CM), given by

$$
\begin{aligned}
\langle\hat{\mathbf{R}}\rangle & =(\langle\hat{x}\rangle,\langle\hat{p}\rangle), \\
{[\sigma]_{k l} } & =\frac{1}{2}\left\langle\left\{\hat{R}_{k}, \hat{R}_{l}\right\}\right\rangle-\left\langle\hat{R}_{k}\right\rangle\left\langle\hat{R}_{l}\right\rangle,
\end{aligned}
$$

where $\hat{\mathbf{R}} \equiv(\hat{x}, \hat{p})$, the anticommutator is denoted as $\{\cdot, \cdot\}$, and the expectation values $\langle\cdot\rangle$ are calculated over $\varrho$ by the Born rule.

A useful measure of $\mathrm{nG}$ for a quantum state may be obtained by introducing a reference Gaussian state $\varrho_{G}$, having the same $\mathrm{CM}$ and first-moment vector of the considered state $\hat{\varrho}$, namely $\left\langle\hat{\mathbf{R}}_{G}\right\rangle=\langle\hat{\mathbf{R}}\rangle$ and $\sigma_{G}=\sigma$. Given the von Neumann entropy $S(\hat{\varrho})=-\operatorname{Tr}[\hat{\varrho} \ln \hat{\varrho}]$, the $n G$ measure is then defined as the quantum relative entropy (QRE) of these two states, i.e., $\delta_{\mathrm{nG}}[\hat{\varrho}] \equiv S\left[\hat{\varrho} \| \hat{\varrho}_{G}\right]=\operatorname{Tr}\left[\hat{\varrho}\left(\ln \hat{\varrho}-\ln \hat{\varrho}_{G}\right)\right]$, which, eventually, reduces to

$$
\delta_{\mathrm{nG}}[\hat{\varrho}]=S\left(\hat{\varrho}_{G}\right)-S(\hat{\varrho})
$$

by exploiting the assumptions made on the reference Gaussian state $\hat{\varrho}_{G}$ [24]. Furthermore, this quantifier corresponds to the relative entropy of $\mathrm{nG}$, i.e., the minimum relative entropy between the state into consideration and the whole set of Gaussian states [37].

The quantum relative entropy is not a proper metric, since it is not symmetric under exchange of its arguments. In spite of this issue, it is a well-defined measure of statistical distinguishability between quantum states, since the probability of confusing two states $\hat{\varrho}_{1}$ and $\hat{\varrho}_{2}$ when performing measurement on a large number $N$ of copies of $\hat{\varrho}_{2}$ is $e^{-N S\left[\rho_{1} \| \rho_{2}\right]}$ [38]. For a single mode Gaussian state the von Neumann entropy is fully determined by its CM as $S\left(\hat{\varrho}_{G}\right)=h(\sqrt{\operatorname{det} \sigma})$, where the function $h(x)$ is given by

$$
h(x)=\left(x+\frac{1}{2}\right) \ln \left(x+\frac{1}{2}\right)-\left(x-\frac{1}{2}\right) \ln \left(x-\frac{1}{2}\right) .
$$

In the following, we will be mostly interested in pure states, for which $S(\hat{\varrho})=0$; the $\mathrm{nG}$ measure thus assumes a simple form

$$
\delta_{\mathrm{nG}}[\hat{\varrho}]=h(\sqrt{\operatorname{det} \sigma}) .
$$

\section{B. Nonclassicality based on Wigner negativity}

Phase-space analysis is at the heart of several approaches to detect and quantify the nonclassical character of quantum states. Different notions of $\mathrm{nC}$ may be introduced, stemming from the quasiprobability distributions associated to the state under scrutiny, or by minimizing the distance to a set of classical states, similar to the $\mathrm{nG}$ measurement we have just introduced.

From the physical point of view, the most relevant notion of nonclassicality is associated to the Glauber-Sudarshan $P$ function. A state is $P$ nonclassical when its $P$ function is not a well-behaved density of probability, i.e., includes singularities and/or negative parts [39-41]. This notion of nonclassicality stems from the fact that coherent states are the only pure quantum states that show classical features from the point of view of quantum optics. A good measure of $P$-nC is the socalled nonclassical depth $[42,43]$, which consists in evaluating the minimal amount of Gaussian noise required to turn the $P$ function into a well-behaved probability distribution. A caveat of this measure is that all pure nG states saturate the upper bound of the nonclassical depth, i.e., they are all maximally nonclassical under this measure [44]. As a consequence this measure is not helpful to analyze in detail the $\mathrm{nC}$ properties of the pure Gaussian states we are going to consider hereafter. This notion of nonclassicality has been recently studied in the context of resource theories [45], where an operational interpretation in terms of metrological usefulness has been introduced [46,47].

An alternative approach, based on Wigner function, has been suggested a few years ago [23]. The $\mathrm{W}$ function is known 
to be a well-defined quasiprobability density function, i.e., it may display negative parts, but it never has singularities. The $W$ nonclassicality $(W-\mathrm{nC})$ corresponds to the negative volume of the Wigner function, and it provides an intuitive and practical way to detect the amount of $\mathrm{nC}$ of a quantum state, as it allows one to distinguish the degree of $\mathrm{nC}$ for different pure states. Moreover, this stronger form of nonclassicality (any $W$ nonclassical state is also $P$ nonclassical, while the opposite is not necessarily true) is the crucial resource for several quantum information tasks (see below).

$W$-nC for a generic single-mode quantum state $\hat{\varrho}$ is defined as follows:

$$
\begin{aligned}
\delta_{\mathrm{nC}}[\hat{\varrho}] & =\int d x d p[|W[\hat{\varrho}](x, p)|-W[\hat{\varrho}](x, p)] \\
& =\int d x d p|W[\hat{\varrho}](x, p)|-1,
\end{aligned}
$$

where the integration is performed over the whole $2 \mathrm{D}$ phase space and the second equality is obtained taking into account the normalization of the Wigner function. Notice that the Wigner function may also be computed directly, without passing through the characteristic function, upon employing the following expression, valid for any $N$-density operator:

$$
W[\hat{\varrho}](\boldsymbol{X})=\frac{2}{\pi} \operatorname{Tr}[\hat{\varrho} \hat{D}(2 \boldsymbol{X}) \hat{\Pi}],
$$

with the parity operator defined as $\hat{\Pi}=(-1)^{\hat{a}^{\dagger} \hat{a}}$ and $\hat{D}(2 \boldsymbol{X})=\bigotimes_{k=1}^{n} \hat{D}\left(2 x_{k}, 2 p_{k}\right)$, where $\hat{D}\left(2 x_{k}, 2 p_{k}\right)=$ $\exp \left\{2 i p_{k} \hat{x}_{k}-2 i x_{k} \hat{p}_{k}\right\}$ is the usual single-mode displacement operator.

From an operational point of view, the notion of $\mathrm{W}-\mathrm{nC}$ has been connected to the impossibility of efficiently simulating a quantum system via phase-space methods [48,49], or more quantitatively to the hardness of estimating the output probabilities of an experiment [50]. In turn, this fact seems to play an important role for schemes aimed at quantum supremacy with homodyne detection [51,52]. For these reasons, W-nC has been studied in the context of an operational resource theory where the free operations are Gaussian ones; a particularly useful monotone is the so-called Wigner logarithmic negativity [27], defined as $\log \left(\delta_{\mathrm{nC}}[\hat{\varrho}]+1\right)$.

We also stress the deep connection between $\mathrm{nG}$ states and $\mathrm{nC}$ states with a negative Wigner function. According to the Hudson theorem [53], Gaussian states are the only pure states with a positive $W$, while for mixed states the situation is more involved [54-56]. Interestingly, for families of pure non-Gaussian states where only a single parameter is varied, quantifiers of $\mathrm{nG}$ and $W-\mathrm{nC}$ were always found to be in a monotonic relationship. In particular, this observation has been made for ground states of anharmonic oscillators [57], where the behavior of the two quantities is also qualitatively very similar. However, note that in some cases the behavior can be wildly different, while retaining monotonicity, e.g., when varying the amplitude of cat states the measure $\delta_{\mathrm{nC}}$ saturates to a finite value, while $\delta_{\mathrm{nG}}$ diverges [27].

Notice also that the quantifier of W-nC in Eq. (6) has the valuable property of being accessible through experimental measurements, since the Wigner function can be reconstructed by means of tomographic techniques, involving photon counting or homodyne detection of the marginal distributions
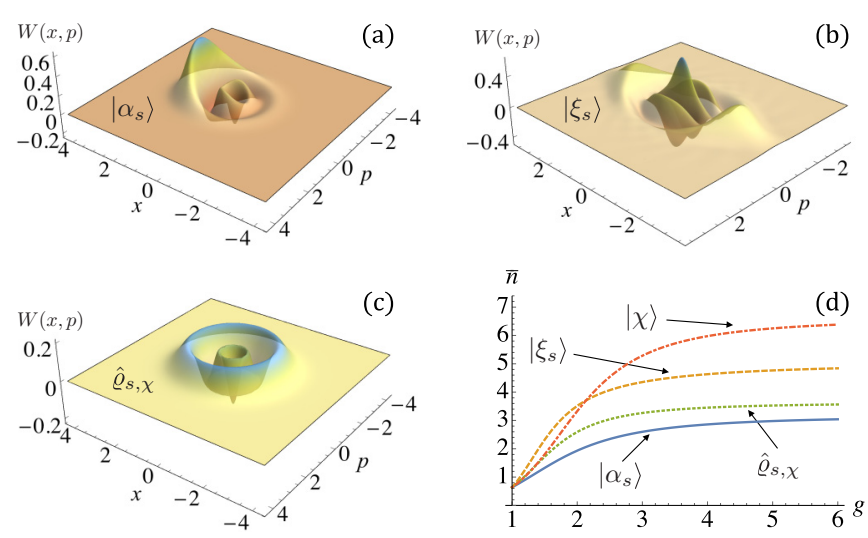

FIG. 1. Wigner functions $W(x, p)$ of (a) the amplified coherent state $\left|\alpha_{s}\right\rangle$ of Eq. (14), (b) the amplified squeezed vacuum state $\left|\xi_{s}\right\rangle$ of Eq. (17), and (c) the reduced state of an amplified twin beam $\hat{\varrho}_{s, \chi}$ of Eq. (21). These amplified states clearly show a highly nonclassical and non-Gaussian behavior. The plots have been obtained by setting (a) $\alpha=0.8$, (b) $\xi=0.73$, (c) $\chi=0.63$, and $g=4, p=3$. (d) The effect of amplification in the average photon number $\bar{n}$ for these states, together with the amplified TWB of Eq. (24), is plotted against the gain parameter $g$, assuming the same average photon number $\bar{n}=0.64$ at $g=1$.

of $W[\hat{\varrho}](x, p)$ [58-61]. Another experimentally friendly quantifier of $W$-nC based on relative entropy was also proposed [62].

In the following sections, we are going to consider different kinds of signals undergoing noiseless amplification. In order to qualitatively anticipate the nature of our results, let us provide a phase-space snapshot of the corresponding conditionally amplified states: in Fig. 1 we show the Wigner functions of an amplified coherent state, an amplified squeezed vacuum state, and the reduced state of an amplified twin beam. The $W$ negativity and the $\mathrm{nG}$ character of the amplified states clearly emerge. We also show the effect of amplification in terms of the output signal energy (i.e., average photon number), assuming the same average photon number $\bar{n}$ at $g=1$.

\section{Noiseless linear amplification}

Ideally, a perfect amplification would be obtained by applying the operator $\hat{T}=g^{\hat{a}^{\dagger} \hat{a}}$, with a positive gain parameter $g>1$. The idea behind the NLA is to approximate the action of this ideal nonunitary operator, by implementing a measurement protocol with postselection [5]. In fact, the impossibility of deterministic noiseless linear amplifiers stems from the unitarity and linearity of quantum evolution that can only be broken by considering conditional evolutions due to measurements. However, it is not even possible to implement the ideal operation with a finite probability of success and there is a trade-off between the probability of success and the fidelity to the desired results. The usual approach to obtain a feasible implementation with nonzero probability of success is to implement the action of the ideal amplification only on a finite-dimensional truncation of the Fock space. An optimal (with respect to the previously mentioned trade-off) measurement (Kraus) operator for this scheme was introduced in [13] and further studied [14]. The action of this protocol 
is described as follows: we consider two possible outcomes, success $(s)$ and failure $(f)$, which correspond to a two-element $\operatorname{POVM}\left\{\hat{E}_{s}^{p^{\dagger}} \hat{E}_{s}^{p}, \hat{E}_{f}^{p^{\dagger}} \hat{E}_{f}^{p}\right\}$ such that $\hat{E}_{s}^{p^{\dagger}} \hat{E}_{s}^{p}+\hat{E}_{f}^{p^{\dagger}} \hat{E}_{f}^{p}=\hat{\mathbb{I}}$. The success measurement operator reads

$$
\hat{E}_{s}^{p}=g^{-p} \sum_{n=0}^{p} g^{n}|n\rangle\left\langle n\left|+\sum_{n=p+1}^{\infty}\right| n\right\rangle\langle n|,
$$

where $p$ is an integer truncation parameter of the Fock space, i.e., the amplification threshold. The probability of a successful amplification when the measurement scheme is applied to a generic pure state $|\psi\rangle$ may be written as

$$
\begin{aligned}
P_{s, \psi} & =\left\langle\psi\left|\hat{E}_{s}^{p^{\dagger}} \hat{E}_{s}^{p}\right| \psi\right\rangle \\
& =g^{-2 p} \sum_{n=0}^{p} g^{2 n}|\langle n \mid \psi\rangle|^{2}+\sum_{n=p+1}^{\infty}|\langle n \mid \psi\rangle|^{2} .
\end{aligned}
$$

The corresponding conditionally amplified state is

$$
\left|\psi_{s}\right\rangle=\frac{\hat{E}_{s}^{p}|\psi\rangle}{\sqrt{P_{s, \psi}}} .
$$

In the limit of low gain, i.e., $g=1+\gamma$ with $0<\gamma \ll 1$, we may write the success operator in the following simplified form:

$$
\hat{E}_{s}^{p} \stackrel{\gamma \ll 1}{\simeq} \mathbb{I}-\gamma \sum_{n=0}^{p}(p-n)|n\rangle\langle n| .
$$

The corresponding probability of amplification may be written as

$$
P_{s, \psi} \stackrel{\gamma \ll 1}{\simeq} 1-2 \gamma \sum_{n=0}^{p}(p-n)|\langle\psi \mid n\rangle|^{2} .
$$

Finally, we remark that, while this optimal NLA scheme has not yet been implemented experimentally, a theoretical implementation obtained by interaction of the light mode with a two-level system was proposed in [14].

In the following, we will consider amplification of paradigmatic examples of Gaussian states, with emphasis on the conditional generation of non-Gaussian and nonclassical amplified states by means of the NLA process.

\section{ENGINEER NON-GAUSSIAN AND NONCLASSICAL STATES BY NOISELESS AMPLIFICATION OF SINGLE-MODE SIGNALS}

\section{A. Noiseless amplification of coherent states}

In order to assess the performances of NLAs in the generation on $\mathrm{nC}$ and $\mathrm{nG}$, let us start by investigating their action on coherent states, which can be easily generated experimentally. Coherent states correspond to displaced vacuum states $\hat{D}(\alpha)|0\rangle$, and may be expressed in the Fock basis as

$$
|\alpha\rangle=e^{-\frac{|\alpha|^{2}}{2}} \sum_{n=0}^{\infty} \frac{\alpha^{n}}{\sqrt{n !}}|n\rangle,
$$

where $\alpha$ is the coherent state amplitude and $\bar{n}=|\alpha|^{2}$ the mean photon number. According to Eq. (10), an amplified coherent
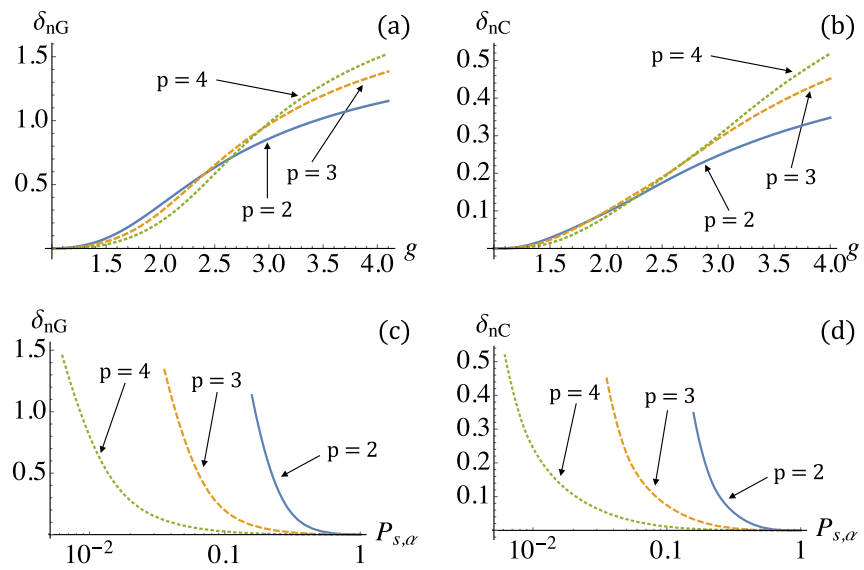

FIG. 2. Non-Gaussianity and nonclassicality of the amplified coherent state of Eq. (14). (a) Plot of the $\mathrm{nG}$ measure $\delta_{\mathrm{nG}}$ as a function of the gain parameter $g$. (b) Plot of the $\mathrm{nC}$ measure $\delta_{\mathrm{nC}}$ as a function of the gain parameter $g$. Plots of $\delta_{\mathrm{nG}}(\mathrm{c})$ and $\delta_{\mathrm{nC}}(\mathrm{d})$ as a function of the success probability $P_{s, \alpha}$. The three curves for each plot correspond to different NLA thresholds: $p=2$ (solid blue), $p=3$ (dashed orange), and $p=4$ (dotted green). The amplitude of the coherent state is $\alpha=0.8$.

state reads

$$
\left|\alpha_{s}\right\rangle=\frac{e^{-\frac{\mid \alpha^{2}}{2}}}{\sqrt{P_{s, \alpha}}}\left(g^{-p} \sum_{n=0}^{p} \frac{(g \alpha)^{n}}{\sqrt{n !}}|n\rangle+\sum_{n=p+1}^{\infty} \frac{\alpha^{n}}{\sqrt{n !}}|n\rangle\right),
$$

where $P_{s, \alpha}$ denotes the probability of a successful amplification

$$
P_{s, \alpha}=e^{-|\alpha|^{2}}\left[g^{-2 p} \sum_{n=0}^{p} \frac{(g|\alpha|)^{2 n}}{n !}+\sum_{n=p+1}^{\infty} \frac{|\alpha|^{2 n}}{n !}\right] .
$$

The $\mathrm{nG}$ of the resulting amplified coherent state is assessed in terms of the measure $\delta_{\mathrm{nG}}$, as described in Sec. II A, and, since the conditional state $\left|\alpha_{s}\right\rangle$ generated by the NLA is pure, it can be easily computed by means of Eq. (5). As shown in the upper left panel of Fig. 2, the action of the nondeterministic NLA on a coherent state is to generate an amplified noiseless pure state (14) with an amount of $\mathrm{nG}$ monotonically increasing with the gain parameter $g$. In particular, we chose, without loss of generality, a real coherent amplitude $\alpha=0.8$ and different values of the threshold parameter $p=2,3,4$. As it is apparent from the plot, we have a monotone behavior with $g$ for any $p$. The larger is $p$, the larger is the $\mathrm{nG}$ at large values of the gain.

The Wigner function of the amplified coherent state $\hat{\varrho}_{s, \alpha}=$ $\left|\alpha_{s}\right\rangle\left\langle\alpha_{s}\right|$ may be conveniently obtained from the expression (7). As it can be appreciated from the plot in Fig. 1(a), the Wigner function clearly has negative values. In the upper right panel of Fig. 2, the $W$-nC of an amplified coherent state, i.e., $\delta_{\mathrm{nC}}\left[\hat{\varrho}_{s, \alpha}\right]$ in Eq. (6), is shown as a function of the gain $g$ of the NLA. We notice that $W$-nC increases monotonically with $g$, and the larger is the threshold, the larger is $\mathrm{nC}$, for large $g$. Remarkably, both the $\mathrm{nG}$ and the $\mathrm{nC}$ share the same qualitative behavior against the NLA parameters, confirming the connection explained in Sec. II B. The behaviors of the two quantities suggest identifying the NLA gain as the parameter 

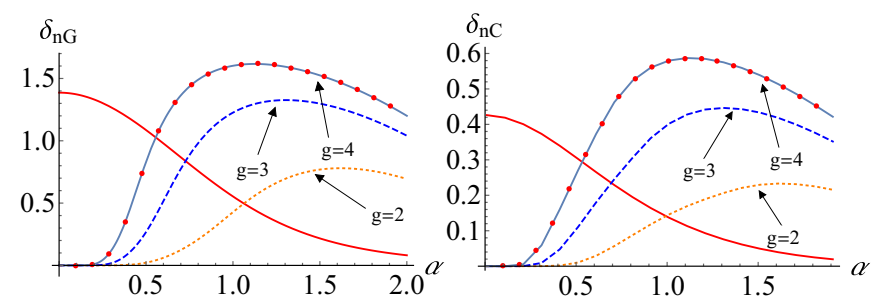

FIG. 3. Plots of the $\mathrm{nG}$ and $\mathrm{nC}$ measures, $\delta_{\mathrm{nG}}$ (left panel) and $\delta_{\mathrm{nC}}$ (right panel), as a function of the input amplitude $\alpha$, for the photon-added coherent state $|\alpha\rangle_{\text {add }}$ (solid red) and the amplified coherent state with different configurations: $g=2$ (dotted orange), $g=3$ (dashed blue), and $g=4$ (red circles). The truncation order $p$ is fixed at 4 in each plot.

driving the semiclassical coherent signal to a highly nonGaussian and nonclassical one. The lower panels of Fig. 2 illustrate the tradeoff between the amount of obtained $\mathrm{nG}$ (nC) and the corresponding probability $P_{s, \alpha}$ of successful amplification. Larger values of $P_{s, \alpha}$ are obtained for smaller gain.

We now compare the performances of our suggested scheme with a well-known de-Gaussification protocol proposed for the generation of non-Gaussian and nonclassical states. In particular, we will consider photon addition performed on coherent states of light. The resulting state, known as photon-added coherent state (PAC), assumes the following expression: $\left|\alpha_{\text {add }}\right\rangle=\mathcal{N}_{\text {add }} \hat{a}^{\dagger}|\alpha\rangle$, where $\mathcal{N}_{\text {add }}=(1+$ $\left.|\alpha|^{2}\right)^{-1 / 2}$. Generally speaking, photon added states can be generated by coupling the input with an ancillary single photon state in a beam splitter, then conditioning the output state upon the outcome "no photons detected." We recall that this class of states is experimentally available $[63,64]$. As the PACs remain pure, their entropic non-Gaussianity $\delta_{\mathrm{nG}}$ is obtained via Eq. (5). In Fig. 3, we compare the $\mathrm{nG}$ and $\mathrm{nC}$ obtained from coherent states, either acting with photon addition or with the NLA for different values of the gain. As it appears, when the input amplitude exceeds a certain critical value $\alpha_{c}$ that depends on the configuration of the NLA, the de-Gaussification protocol based on the NLA outperforms photon addition. This observation suggests that the NLA may be a powerful resource for non-Gaussianity when applied to states outside the disk of radius $|\alpha| / g$, as previously pointed out. As one may expect, for larger value of the gain $g$, the critical value $\alpha_{c}$ becomes smaller. Our comparison of both the entropic non-Gaussianity and the $\mathrm{W}$ nonclassicality thus testifies the robustness of the optimal NLA for engineering highly non-Gaussian and nonclassical states.

\section{B. Noiseless amplification of squeezed vacuum}

Another important Gaussian state, employed as a resource in many quantum protocols, is the squeezed vacuum state $|\xi\rangle=\hat{\mathcal{S}}(\xi)|0\rangle$, where the squeezing operator $\hat{\mathcal{S}}(\xi)=$ $\exp \left\{\frac{1}{2} \xi\left(\hat{a}^{\dagger}\right)^{2}-\frac{1}{2} \xi^{*}(\hat{a})^{2}\right\}$ acts on the vacuum state. The phase of the squeezing parameter $\xi=r e^{i \phi}$ specifies which quadrature of the field is squeezed, whereas its modulus quantifies the amount of squeezing. The expression of a squeezed
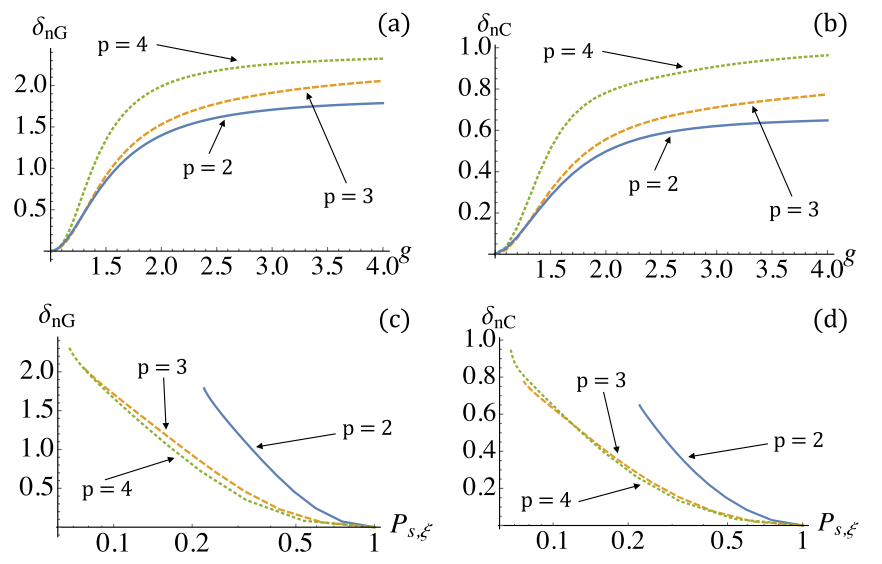

FIG. 4. Non-Gaussianity and nonclassicality of the amplified squeezed vacuum state of Eq. (17). (a) $\mathrm{nG}$ measure $\delta_{\mathrm{nG}}$ plotted against the gain parameter $g$. (b) Plot of the $\mathrm{nC}$ measure $\delta_{\mathrm{nC}}$ as a function of the gain $g$. Plots of $\delta_{\mathrm{nG}}(\mathrm{c})$ and $\delta_{\mathrm{nC}}(\mathrm{d})$ as a function of the success probability $P_{s, \xi}$. The three curves for each plot correspond to different NLA thresholds: $p=2$ (solid blue), $p=3$ (dashed orange), and $p=4$ (dotted green). The squeezing parameter is set to $r=0.73$.

vacuum in the Fock basis is given by

$$
\begin{aligned}
|\xi\rangle & =\frac{1}{\sqrt{\mu}} \sum_{n=0}^{\infty}\left(\frac{v}{2 \mu}\right)^{n} \frac{\sqrt{(2 n) !}}{n !}|2 n\rangle \\
& \equiv \sum_{n=0}^{\infty} x_{n}|2 n\rangle, \quad \sum_{n=0}^{\infty}\left|x_{n}\right|^{2}=1,
\end{aligned}
$$

where $\mu=\cosh r$ and $v=e^{i \phi} \sinh r$. The action of NLA on the squeezed vacuum with a successful amplification, employing Eq. (10), reads

$$
\left|\xi_{s}\right\rangle=\frac{1}{\sqrt{P_{s, \xi}}}\left(g^{-p} \sum_{\substack{n=0 \\(n \text { even })}}^{p} g^{n} x_{\frac{n}{2}}|n\rangle+\sum_{\substack{n=p+1 \\(n \text { even })}}^{\infty} x_{\frac{n}{2}}|n\rangle\right)
$$

where the success probability is given by

$$
P_{s, \xi}=g^{-2 p} \sum_{n=0}^{p} g^{2 n}\left|x_{\frac{n}{2}}\right|^{2}+\sum_{n=p+1}^{\infty}\left|x_{\frac{n}{2}}\right|^{2} .
$$

In the upper left panel of Fig. 4, we show the nG measure $\delta_{\mathrm{nG}}\left[\hat{\varrho}_{s, \xi}\right]$ for the amplified state $\hat{\varrho}_{s, \xi}=\left|\xi_{s}\right\rangle\left\langle\xi_{s}\right|$ as a function of the gain parameter $g$, for different values of the threshold $p=2,3,4$, at a fixed value of the squeezing parameter $\xi=$ 0.73 , corresponding to the same input energy, at $g=1$, of a coherent state with $\alpha=0.8$. We notice that, starting from a squeezed vacuum, the efficiency of the NLA in generating non-Gaussianity is much larger than in the coherent-state case, in particular for low values of the gain parameter.

In the upper right panel of Fig. 4, we show the nonclassicality $\delta_{\mathrm{nC}}\left[\hat{\varrho}_{s, \xi}\right]$ as a function of the gain parameter $g$, for different values of the threshold $p=2,3,4$, at a fixed value of the squeezing strength $r=0.73$. Similar to the $\mathrm{nG}$ measure, 
amplification generates a large amount of nonclassicality, also for low values of the gain. The role of the threshold $p$, likewise, is to increment the $\mathrm{nC}$ measure. Again, $\delta_{\mathrm{nG}}\left[\hat{\varrho}_{s, \xi}\right]$ and $\delta_{\mathrm{nC}}\left[\hat{\varrho}_{s, \xi}\right]$ are two increasing monotonic functions, and the amplified state is highly nonclassical and non-Gaussian at the same time. The lower panels of Fig. 4 illustrate the trade-off between the amount of $\mathrm{nG}(\mathrm{nC})$ obtained and the success probability $P_{s, \alpha}$. Remarkably, increasing the threshold value from $p=3$ to $p=4$ leads to substantially larger values of $\mathrm{nG}$ and $\mathrm{nC}$, with basically the same success probability. Furthermore, for the value $p=2$ significant values of the $\mathrm{nG}$ and the $\mathrm{nC}$ are achieved with interesting success rates. Hence highly non-Gaussian and nonclassical states may be engineered through the action of an optimal NLA with appealing efficiency.

\section{ENGINEER NON-GAUSSIAN AND NONCLASSICAL STATES BY NOISELESS AMPLIFICATION OF TWIN BEAM}

\section{A. Destructive noiseless amplification of twin beam}

The projection postulate offers a viable mechanism to realize synthetic dynamics and, in turn, to generate quantum states otherwise unreachable with Hamiltonian evolution [18]. In this section, we analyze the effects of both destructive and nondestructive implementations of NLA on maximally entangled continuous-variable states, namely the twin-beam (TWB) state:

$$
|\chi\rangle=\sqrt{1-\chi^{2}} \sum_{n=0}^{\infty} \chi^{n}|n, n\rangle,
$$

where $0<\chi<1$ and $|n, n\rangle=|n\rangle \otimes|n\rangle$ is the Fock basis for the two-mode system. The TWB state is a Gaussian twomode state obtained by the action of the two-mode squeezing operator $\hat{\mathcal{S}}_{2}(\chi)=\exp \left\{\chi \hat{a} \hat{b}-\chi^{*} \hat{a}^{\dagger} \hat{b}^{\dagger}\right\}$ on the vacuum $|0,0\rangle$, where $a$ and $b$ denote the two involved modes. Without loss of generality, we will consider $\chi$ as a real parameter. These states of light may be generated in nondegenerate optical parametric amplifiers by spontaneous down-conversion or by mixing at a balanced linear mixer two squeezed vacua with opposite squeezing phases. Since the efficiencies of these processes are relatively weak, it is of interest to investigate protocols to enhance the resulting nonclassical properties.

According to the reduction postulate, a measurement performed on one of the two constituents of an entangled bipartite system leaves the other part in a conditional state, which depends on the outcome of the measurement. Considering the twin-beam state (19) and a successful NLA amplification performed on subsystem $a$, the subsystem $b$ is reduced into a diagonal state

$$
\begin{aligned}
\hat{\varrho}_{s, \chi} & =\frac{1}{P_{s, \chi}} \operatorname{Tr}_{a}\left[|\chi\rangle\langle\chi| \hat{E}_{s}^{p^{\dagger}} \hat{E}_{s}^{p} \otimes \hat{\mathbb{I}}_{b}\right] \\
& =\frac{\left(1-\chi^{2}\right)}{P_{s, \chi}}\left[g^{-2 p} \sum_{n=0}^{p}(g \chi)^{2 n}|n\rangle\left\langle n\left|+\sum_{n=p+1}^{\infty} \chi^{2 n}\right| n\right\rangle\langle n|\right],
\end{aligned}
$$


FIG. 5. Non-Gaussianity and nonclassicality of the amplified state of Eq. (21). (a) $\mathrm{nG}$ measure $\delta_{\mathrm{nG}}$ plotted against the gain parameter $g$. (b) Plot of the $\mathrm{nC}$ measure $\delta_{\mathrm{nC}}$ as a function of the gain $g$. Plots of $\delta_{\mathrm{nG}}(\mathrm{c})$ and $\delta_{\mathrm{nC}}(\mathrm{d})$ as a function of the success probability $P_{s, \chi}$. The three curves for each plot correspond to different thresholds $p=2$ (solid blue), $p=3$ (dashed orange), and $p=4$ (dotted green). The TWB parameter is set to $\chi=0.63$.

where $\hat{E}_{s}^{p \dagger} \hat{E}_{s}^{p}$ denotes an element of the POVM described by the NLA, $\hat{\mathbb{I}}_{b}$ is the identity operator acting on $\mathcal{H}_{b}$, and $P_{s, \chi}$ is the probability of successful amplification:

$$
P_{s, \chi}=\left(1-\chi^{2}\right)\left[g^{-2 p} \sum_{n=0}^{p}(g \chi)^{2 n}+\sum_{n=p+1}^{\infty} \chi^{2 n}\right] .
$$

In order to compute the $\mathrm{nG}$ measure for the amplified state in $\hat{\varrho}_{s, \chi}$ we refer to Eq. (3). As the state (21) is diagonal in the Fock basis, its reference Gaussian state $\hat{\varrho}_{G}$ is a thermal state with mean photon number $\bar{n}_{s, \chi}=\operatorname{Tr}\left[\hat{\varrho}_{s, \chi} \hat{a}^{\dagger} \hat{a}\right]$ and diagonal $\mathrm{CM} \sigma=\frac{1}{2} \operatorname{Diag}\left(1+2 \bar{n}_{s, \chi}, 1+2 \bar{n}_{s, \chi}\right)$. Moreover, the von Neumann entropy $S\left(\hat{\varrho}_{s, \chi}\right)=-\sum_{n} \rho_{n} \ln \rho_{n}$ can be directly calculated with the diagonal matrix elements $\rho_{n}$ of the state (21). The resulting $\mathrm{nG}$ measure for this amplified mixed state can, thus, be written in this simple form:

$$
\delta_{\mathrm{nG}}\left[\hat{\varrho}_{s, \chi}\right]=h\left(\frac{1}{2}+\bar{n}_{s, \chi}\right)+\sum_{n} \rho_{n} \ln \rho_{n} .
$$

In the upper left panel of Fig. 5 we show the nG measure (23) as a function of the gain parameter $g$ for the amplified state $\hat{\varrho}_{s, \chi}$. The three curves correspond to different thresholds $p=2,3,4$ and, as already observed in the previous cases, the function $\delta_{\mathrm{nG}}\left[\hat{\varrho}_{s, \chi}\right]$ increases monotonically with the gain. The same happens if one increases the threshold.

As highlighted in Fig. 1(c), the Wigner function of the amplified state $\hat{\varrho}_{s, \chi}$ may assume negative values. The $\mathrm{nC}$ measure $\delta_{\mathrm{nC}}\left[\hat{\varrho}_{s, \chi}\right]$ is shown as a function of the gain $g$ in the upper right panel of Fig. 5 and it displays a monotonic growth. At variance with the previous cases, i.e., amplification of coherent and squeezed vacuum states, we observe that the W-nC of $\hat{\varrho}_{s, \chi}$ is zero before a threshold value of the gain, and then starts to grow monotonically for any value of $p$. This happens since the Wigner function of the amplified state is diagonal in the Fock basis and, thus, phase independent. It begins to warp as the gain increases but remains positive. Only after a particular 

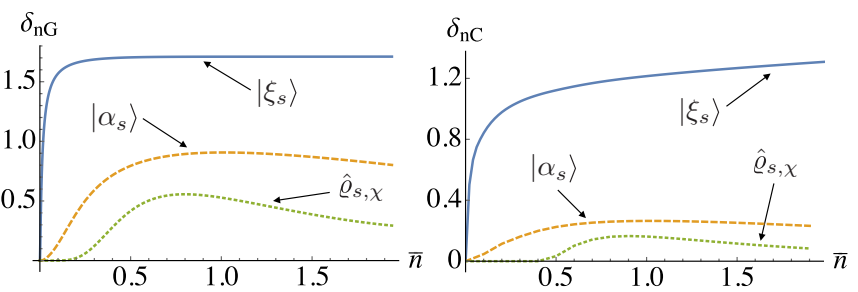

FIG. 6. Plots of the $\mathrm{nG}$ and $\mathrm{nC}$ measures, $\delta_{\mathrm{nG}}$ (left panel) and $\delta_{\mathrm{nC}}$ (right panel), as a function of the mean input energy $\bar{n}$, for the amplified states $\left|\alpha_{s}\right\rangle$ (dashed orange), $\left|\xi_{s}\right\rangle$ (solid blue), and $\hat{\varrho}_{s, \chi}$ (dotted green). The parameters of the NLA, $g=3$ and $p=2$, are fixed in each plot.

value of the gain, depending mainly on the TWB parameter $\chi$, the Wigner function takes on negative volumes. Thus we have found that the two quantifiers we considered are still in a monotonic relationship, even though they have a qualitatively different behavior, as already mentioned in Sec. II B.

Let us now compare the performances of the amplification process in the generation of single-mode $\mathrm{nG}$ and $\mathrm{nC}$. To this purpose, we consider input signals with the same initial energy undergoing identical amplification processes, i.e., $\bar{n}_{\alpha}=\bar{n}_{\xi}=\bar{n}_{\chi} \equiv \bar{n}$, where $\bar{n}_{\alpha} \equiv\left\langle\alpha\left|\hat{a}^{\dagger} \hat{a}\right| \alpha\right\rangle=|\alpha|^{2}, \bar{n}_{\xi} \equiv$ $\left\langle\xi\left|\hat{a}^{\dagger} \hat{a}\right| \xi\right\rangle=\sinh ^{2} r$, and $\bar{n}_{\chi}=\chi^{2} /\left(1-\chi^{2}\right)$. In Fig. 6 we plot $\delta_{\mathrm{nG}}$ (left panel) and $\delta_{\mathrm{nC}}$ (right panel) as a function of $\bar{n}$, with fixed NLA parameters $g=3$ and $p=2$. In particular, we see that for both the $\mathrm{nG}$ and $\mathrm{nC}$ measures the amplified squeezed vacuum state (17) performs better than the amplified coherent state (14), whereas the amplified reduced TWB state (21) has the lowest values of $\mathrm{nG}$ and $\mathrm{W}-\mathrm{nC}$. We observe that the single-mode squeezing is a better resource in obtaining highly non-Gaussian and nonclassical states, whereas the destructive measurement performed on the amplified TWB state somehow spoils the input quantumness.

\section{B. Increasing entanglement by nondestructive NLA on twin beam}

In this section, we examine the action of a successful nondestructive probabilistic amplification on a TWB state by focusing on the $\mathrm{nG}$ and the entanglement between the two correlated modes. Applying the NLA on one mode of a TWB results in the following pure conditional state:

$$
\begin{aligned}
\left|\chi_{s}\right\rangle & =\frac{\hat{E}_{s}^{p} \otimes \mathbb{I}_{b}|\chi\rangle}{\sqrt{P_{s, \chi}}} \\
& =\frac{\sqrt{1-\chi^{2}}}{\sqrt{P_{s, \chi}}}\left(g^{-p} \sum_{n=0}^{p}(g \chi)^{n}|n, n\rangle+\sum_{n=p+1}^{\infty} \chi^{n}|n, n\rangle\right),
\end{aligned}
$$

where the success probability is given by Eq. (22). The $\mathrm{nG}$ measure for the amplified TWB state reduces to Eq. (5), as the state (24) remains pure under the action of the NLA. In order to compute the reference Gaussian state, we make some considerations on the CM (2b) of a two-mode Gaussian state $\sigma_{2}$, which can be written in a block form as

$$
\sigma_{2}=\left(\begin{array}{cc}
\boldsymbol{A} & \boldsymbol{C} \\
\boldsymbol{C}^{T} & \boldsymbol{B}
\end{array}\right),
$$

where $\boldsymbol{A}, \boldsymbol{B}$, and $\boldsymbol{C}$ are $2 \times 2$ matrices. By means of local symplectic transformations, it is possible to derive four symplectic invariants, namely $I_{1} \equiv \operatorname{det} \boldsymbol{A}, I_{2} \equiv \operatorname{det} \boldsymbol{B}, I_{3} \equiv \operatorname{det} \boldsymbol{C}$, and $I_{4} \equiv \operatorname{det} \sigma_{2}$, and derive a simple expression for the two symplectic eigenvalues of $\sigma_{2}$ :

$$
d_{ \pm}=\sqrt{\frac{\Delta\left(\sigma_{2}\right) \pm \sqrt{\Delta^{2}\left(\sigma_{2}\right)-4 I_{4}}}{2}}
$$

where $\Delta\left(\sigma_{2}\right) \equiv I_{1}+I_{2}+2 I_{3}$. The von Neumann entropy of a generic two-mode Gaussian state can be written as $S(\hat{\varrho})=h\left(d_{+}\right)+h\left(d_{-}\right)$. In the case of the amplified TWB state (24) it is easy to see that $I_{1}=I_{2}=\left(\frac{1}{2}+\bar{N}_{\chi}\right)^{2}$, with $\bar{N}_{\chi}=\operatorname{Tr}\left[|\chi\rangle\langle\chi|\left(\hat{a}^{\dagger} \hat{a}+\hat{b}^{\dagger} \hat{b}\right)\right]$, and that $d_{+}=d_{-}=\sqrt{I_{1}+I_{3}}$. Overall, the $\mathrm{nG}$ measure may be expressed as

$$
\delta_{\mathrm{nG}}\left[\left|\chi_{s}\right\rangle\left\langle\chi_{s}\right|\right]=2 h\left(d_{+}\right)=2 \sqrt{I_{1}+I_{3}}
$$

and it is plotted as a function of the gain parameter in the left panel of Fig. 8.

As it is apparent from the plot, we recover the same behavior as in the previous examples concerning the monotonic growth with increasing gain of NLA. We compare the $\mathrm{nG}$ measure of the amplified states (21) and (24) for a NLA with, respectively, destructive and nondestructive measurements. In particular, by fixing the same squeezing parameter $\chi_{1}=0.63$ at $g=1$, meaning that the resource TWB state (19) is fixed, we notice an enhancement of the NLA protocol in the nondestructive-measurement case (dashed curve with respect to the solid one). On the other hand, another comparison can be made by limiting the amount of mean energy of the resource state at $g=1$, i.e., fixing $\bar{n}_{\chi_{1}}=\bar{N}_{\chi_{2}}$ with $\chi_{2}=0.50$. Also, in this case, the enhancement of the amplification process occurs for a NLA with nondestructive measurement (dotted curve with respect to the solid one). The initial resources being equal, a NLA with nondestructive measurement acting on a TWB state strongly enhances the non-Gaussianity character of the amplified state.

Let us compare the amount of non-Gaussianity generated by the NLA with alternative de-Gaussification protocols performed on the TWB state. In particular, we consider photon addition and photon subtraction schemes that are experimentally accessible. In existing experiments, the photon-subtracted squeezed vacuum (PSSV) states are generated from twin beams with beam splitters of low reflexivity, whereas the photon-added squeezed vacuum (PASV) requires weak-gain parametric down converters. The expressions of PSSV and PASV are respectively given by

$$
\begin{gathered}
\left|\chi_{s}\right\rangle=\mathcal{N}_{s} \hat{a} \hat{b}|\chi\rangle=\sqrt{\frac{\left(1-\chi^{2}\right)^{3}}{1+\chi^{2}}} \sum_{n=0}^{\infty} \chi^{n}(n+1)|n, n\rangle, \\
\left|\chi_{a}\right\rangle=\mathcal{N}_{a} \hat{a}^{\dagger} \hat{b}^{\dagger}|\chi\rangle=\sqrt{\frac{\left(1-\chi^{2}\right)^{3}}{1+\chi^{2}}} \sum_{n=0}^{\infty} \chi^{n}(n+1)|n+1, n+1\rangle .
\end{gathered}
$$



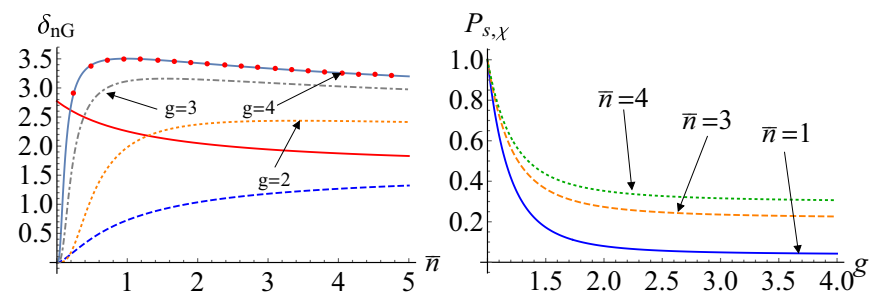

FIG. 7. Left panel: plots of the entropic non-Gaussianity $\delta_{n G}$ as a function of the input energy $\bar{n}$ for the photon-added twin beam (solid red), the photon-subtracted twin beam (dotted blue), and the amplified two-mode squeezed vacuums with different calibrations of the NLA: $g=2$ (dashed orange), $g=3$ (dot-dashed gray), and $g=4$ (red circles). Right panel: the probability of success $P_{s, \chi}$ as a function of the gain parameter $g$ for different input energies of the initial twin beam: $\bar{n}=1$ (solid blue), $\bar{n}=3$ (dashed orange), and $\bar{n}=4$ (dotted green). The truncation order is set on $p=3$ for all the plots.

Since both these states are pure, their non-Gaussianity captured by the quantum relative entropy $\delta_{n G}$ is easily evaluated. In the left panel of Fig. 7, we show plots of the entropic nonGaussianity for the PSSV, the PASV, and the amplified TWB for different configurations as a function of the input energy $\bar{n}$. We observe that, apart from the case of weak input energies, the NLA generates the greatest amount of non-Gaussianity. Moreover, as it appears, the more intense is the amplification, the broadest is the range of input energies where a substantial non-Gaussian character is achieved. In a conditional evolution, the efficiency of the process is determined by the probability of the postselected outcome to occur. Regarding the NLA based scheme, this latter coincides with the probability of a successful amplification. In the right panel of Fig. 7, we plot the rate of success for an amplification to be implemented on twin beams of different energies as a function of the gain (the threshold being fixed at $p=3$ ). We notice that beyond $g=2$, the probability to achieve a successful amplification decreases softly and still attains attractive values notably for high input energies. Interestingly, those configurations where the optimal NLA is efficient lead up to highly non-Gaussian two-mode states, thus identifying its action on relevant input twin beams as a powerful de-Gaussification protocol.

Finally, let us study whether the NLA enhances entanglement in the amplified TWB state. The most notable measure of entanglement for a bipartite pure state $|\Psi\rangle$ is the entropy of entanglement, which is defined as the von Neumann entropy of one of the reduced states $\varrho_{b}=\operatorname{Tr}_{a}[|\Psi\rangle\langle\Psi|]$ :

$$
E[|\Psi\rangle]=S\left[\hat{\varrho}_{b}\right]=-\sum_{n=0}^{\infty} \rho_{n} \ln \rho_{n},
$$

where $\rho_{n}$ are the eigenvalues of the reduced state. In our case, the initial TWB state is pure and the corresponding reduced state of mode $b$ is exactly the amplified state (21) with a nondestructive measurement. We already calculated $S\left(\hat{\varrho}_{s, \chi}\right)$ in the evaluation of the $\mathrm{nG}$ measure $\delta_{\mathrm{nG}}\left[\hat{\varrho}_{s, \chi}\right]$, highlighting the strong relation between non-Gaussianity of the reduced amplified state $\hat{\varrho}_{s, \chi}$ and the amount of entanglement of the bipartite amplified state $\left|\chi_{s}\right\rangle$. We can observe in the right panel of Fig. 8 that the entanglement measure $E\left[\left|\chi_{s}\right\rangle\right]$ shows a
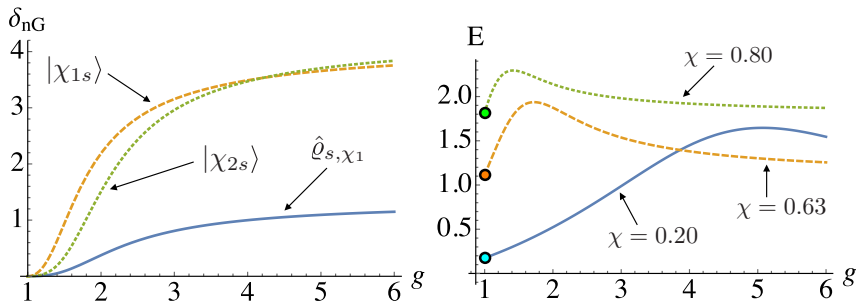

FIG. 8. Left panel: $n G$ measure $\delta_{\mathrm{nG}}$ plotted as a function of the gain parameter $g$ for the amplified states $\hat{\varrho}_{s, \chi_{1}}$ (solid blue curve), $\left|\chi_{1 s}\right\rangle$ and $\left|\chi_{2 s}\right\rangle$ (dashed orange and dotted green curves), with $\chi_{1}=$ 0.63 and $\chi_{2}=0.50$. Right panel: plot of entropy of entanglement $E[|\chi\rangle\langle\chi|]$ as a function of the gain parameter $g$. The three curves correspond to different squeezing parameters of the TWB state: $\chi=$ 0.20 (solid blue), $\chi=0.63$ (dashed orange), and $\chi=0.80$ (dotted green). The three points at $g=1$ are the reference values of the entropy of entanglement for three initial TWB states corresponding to the three values of $\chi$ listed above.

characteristic peak depending on the value of the squeezing parameter, occurring at lower values of the NLA gain for higher values of $\chi$. The remarkable result is provided by the comparison between the curves and the reference values of the amount of entanglement for the initial TWB state at $g=1$ (highlighted points in the figure). For any value of the NLA gain $g>1$, the amplification process brings along an enhancement of entanglement in the amplified state with respect to the amount of entanglement of the corresponding TWB state.

\section{CONCLUSION}

In this work we have investigated the action of a nondeterministic noiseless linear amplifier on single- and two-mode Gaussian states in order to generate highly non-Gaussian and nonclassical amplified quantum states. In particular, we have focused on amplification of feasible Gaussian states, e.g., coherent states, squeezed vacuum states, and entangled twin beam.

Our results show that noiseless amplification is, in general, a powerful scheme to generate nonclassical non-Gaussian states, with the detailed performances depending on the interplay between the gain of the NLA, its threshold for amplification, and the parameters of the input signal. Upon comparing results for input signals with the same initial energy, we have shown that better performances, i.e., larger output $\mathrm{nG}$ and $\mathrm{nC} \mathrm{nG}$, are obtained by amplification of squeezed vacuum. We have also analyzed the performances of nondestructive amplification, showing that amplification of twin beam highly increases entanglement.

Concerning the efficiency of the process, we have shown that, while the probability of successful amplification decreases with the NLA gain, there is a convenient trade-off between $\mathrm{nG}$ (or $\mathrm{nC}$ ) and the success probability itself. We have also shown that for squeezed vacuum input, one may increase $\mathrm{nG}$ and $\mathrm{nC}$ at fixed success probability by increasing the NLA amplification threshold. 
Overall, we have proved that NLA provides an effective scheme to generate highly non-Gaussian and nonclassical states and may be used to increase entanglement in continuous-variable systems. Our results pave the way for optimized implementations of NLA and suggest that both destructive and nondestructive implementations of NLA would be of interest in quantum technology.

\section{ACKNOWLEDGMENTS}

This work has been supported by SERB through Project No. VJR/2017/000011 and by JSPS through Project No. FY2017-S17118. M.G.A.P. is a member of GNFM-INdAM. F.A. acknowledges support from the UK National Quantum Technologies Programm (No. EP/M013243/1). The authors thank Luigi Seveso for several useful discussions.
[1] V. V. Dodonov, J. Opt. B 4, R1 (2002).

[2] A. Serafini, M. G. A. Paris, F. Illuminati, and S. De Siena, J. Opt. B 7, R19 (2005).

[3] K. Hammerer, C. Genes, D. Vitali, P. Tombesi, G. J. Milburn, C. Simon, and D. Bouwmeester, in Cavity Optomechanics, edited by M. Aspelmeyer, T. J. Kippenberg, and F. Marquardt (Springer, Berlin, 2013).

[4] C. M. Caves, Phys. Rev. D 26, 1817 (1982).

[5] T. C. Ralph and A. P. Lund, in Quantum Commun. Meas. Comput., edited by A. Lvovsky (AIP, Melille, NY, 2009), pp. $155-160$.

[6] G. Y. Xiang, T. C. Ralph, A. P. Lund, N. Walk, and G. J. Pryde, Nat. Photon. 4, 316 (2010).

[7] N. Walk, T. C. Ralph, T. Symul, and P. K. Lam, Phys. Rev. A 87, 020303(R) (2013).

[8] F. Ferreyrol, R. Blandino, M. Barbieri, R. Tualle-Brouri, and P. Grangier, Phys. Rev. A 83, 063801 (2011).

[9] F. Ferreyrol, M. Barbieri, R. Blandino, S. Fossier, R. TualleBrouri, and P. Grangier, Phys. Rev. Lett. 104, 123603 (2010).

[10] P. Marek and R. Filip, Phys. Rev. A 81, 022302 (2010).

[11] J. Combes, N. Walk, A. P. Lund, T. C. Ralph, and C. M. Caves, Phys. Rev. A 93, 052310 (2016).

[12] A. Zavatta, J. Fiurášek, and M. Bellini, Nat. Photon. 5, 52 (2011).

[13] S. Pandey, Z. Jiang, J. Combes, and C. M. Caves, Phys. Rev. A 88, 033852 (2013).

[14] N. A. McMahon, A. P. Lund, and T. C. Ralph, Phys. Rev. A 89, 023846 (2014).

[15] T. C. Ralph, A. Gilchrist, G. J. Milburn, W. J. Munro, and S. Glancy, Phys. Rev. A 68, 042319 (2003).

[16] E. Knill, R. Laflamme, and G. J. Milburn, Nature (London) 409, 46 (2001).

[17] T. C. Ralph, A. P. Lund, and H. M. Wiseman, J. Opt. B 7, S245 (2005).

[18] M. G. A. Paris, M. Cola, and R. Bonifacio, Phys. Rev. A 67, 042104 (2003).

[19] A. Kozhekin, G. Kurizki, and B. Sherman, Phys. Rev. A 54, 3535 (1996).

[20] J. Laurat, T. Coudreau, N. Treps, A. Maître, and C. Fabre, Phys. Rev. Lett. 91, 213601 (2003).

[21] M. G. Genoni, F. A. Beduini, A. Allevi, M. Bondani, S. Olivares, and M. G. A. Paris, Phys. Scr. T140, 014007 (2010).

[22] F. Arzani, N. Treps, and G. Ferrini, Phys. Rev. A 95, 052352 (2017).

[23] A. Kenfack and K. Życzkowski, J. Opt. B 6, 396 (2004).

[24] M. G. Genoni, M. G. A. Paris, and K. Banaszek, Phys. Rev. A 78, 060303(R) (2008).
[25] M. G. Genoni and M. G. A. Paris, Phys. Rev. A 82, 052341 (2010).

[26] Q. Zhuang, P. W. Shor, and J. H. Shapiro, Phys. Rev. A 97, 052317 (2018).

[27] F. Albarelli, M. G. Genoni, M. G. A. Paris, and A. Ferraro, Phys. Rev. A 98, 052350 (2018).

[28] R. Takagi and Q. Zhuang, Phys. Rev. A 97, 062337 (2018).

[29] J. Park, J. Lee, K. Baek, S.-w. Ji, and H. Nha, arXiv: 1809.02999.

[30] W. P. Schleich, Quantum Optics in Phase Space (Wiley-VCH, Berlin, New York, 2001).

[31] K. E. Cahill and R. J. Glauber, Phys. Rev. 177, 1882 (1969).

[32] S. M. Barnett and P. M. Radmore, Methods in Theoretical Quantum Optics (Oxford University Press, Oxford, New York, 1997).

[33] A. Ferraro, S. Olivares, and M. G. A. Paris, Gaussian States in Continuous Variable Quantum Information (Bibliopolis, Napoli, 2005).

[34] G. Adesso, S. Ragy, and A. R. Lee, Open Syst. Inf. Dyn. 21, 1440001 (2014).

[35] S. Olivares, Eur. Phys. J. Spec. Top. 203, 3 (2012).

[36] A. Serafini, Quantum Continuous Variables: A Primer of Theoretical Methods (CRC Press, Boca Raton, 2017).

[37] P. Marian and T. A. Marian, Phys. Rev. A 88, 012322 (2013).

[38] V. Vedral, Rev. Mod. Phys. 74, 197 (2002).

[39] R. J. Glauber, Phys. Rev. 130, 2529 (1963).

[40] L. Mandel, Phys. Scr. T12, 34 (1986).

[41] A. Ferraro and M. G. A. Paris, Phys. Rev. Lett. 108, 260403 (2012).

[42] C. T. Lee, Phys. Rev. A 41, 1721 (1990).

[43] C. T. Lee, Phys. Rev. A 44, R2775 (1991).

[44] N. Lütkenhaus and S. M. Barnett, Phys. Rev. A 51, 3340 (1995).

[45] K. C. Tan, T. Volkoff, H. Kwon, and H. Jeong, Phys. Rev. Lett. 119, 190405 (2017).

[46] H. Kwon, K. C. Tan, T. Volkoff, and H. Jeong, Phys. Rev. Lett. 122, 040503 (2019).

[47] B. Yadin, F. C. Binder, J. Thompson, V. Narasimhachar, M. Gu, and M. S. Kim, Phys. Rev. X 8, 041038 (2018).

[48] A. Mari and J. Eisert, Phys. Rev. Lett. 109, 230503 (2012).

[49] V. Veitch, N. Wiebe, C. Ferrie, and J. Emerson, New J. Phys. 15, 013037 (2013).

[50] H. Pashayan, J. J. Wallman, and S. D. Bartlett, Phys. Rev. Lett. 115, 070501 (2015).

[51] T. Douce, D. Markham, E. Kashefi, E. Diamanti, T. Coudreau, P. Milman, P. van Loock, and G. Ferrini, Phys. Rev. Lett. 118, 070503 (2017).

[52] L. Chakhmakhchyan and N. J. Cerf, Phys. Rev. A 96, 032326 (2017) 
[53] R. L. Hudson, Rep. Math. Phys. 6, 249 (1974).

[54] T. Bröcker and R. F. Werner, J. Math. Phys. 36, 62 (1995).

[55] A. Mandilara, E. Karpov, and N. J. Cerf, Phys. Rev. A 79, 062302 (2009).

[56] C. N. Hughes, M. G. Genoni, T. Tufarelli, M. G. A. Paris, and M. S. Kim, Phys. Rev. A 90, 013810 (2014).

[57] F. Albarelli, A. Ferraro, M. Paternostro, and M. G. A. Paris, Phys. Rev. A 93, 032112 (2016).

[58] K. Banaszek, C. Radzewicz, K. Wódkiewicz, and J. S. Krasiński, Phys. Rev. A 60, 674 (1999).

[59] W. Vogel, Phys. Rev. Lett. 84, 1849 (2000).
[60] A. I. Lvovsky and J. H. Shapiro, Phys. Rev. A 65, 033830 (2002).

[61] A. Allevi, A. Andreoni, M. Bondani, G. Brida, M. Genovese, M. Gramegna, P. Traina, S. Olivares, M. G. A. Paris, and G. Zambra, Phys. Rev. A 80, 022114 (2009).

[62] A. Mari, K. Kieling, B. Melholt Nielsen, E. S. Polzik, and J. Eisert, Phys. Rev. Lett. 106, 010403 (2011).

[63] A. Zavatta, S. Viciani, and M. Bellini, Science 306, 660 (2004).

[64] M. Barbieri, N. Spagnolo, M. G. Genoni, F. Ferreyrol, R. Blandino, M. G. A. Paris, P. Grangier, and R. Tualle-Brouri, Phys. Rev. A 82, 063833 (2010). 